\title{
Recurrent Malignant Phylloides Tumor: A Rare Entity
}

Malik $\mathbf{S}^{*}$, Gupta S, Singh S, Sharma J and Sen R

Department of Pathology, Pandit Bhagwat Dayal Sharma Post Graduate Institute of Medical Sciences, Rohtak, Haryana, India

*Corresponding author: Malik S, Resident, Department of Pathology, Pandit Bhagwat Dayal Sharma Post Graduate Institute of Medical Sciences, Rohtak, Haryana, India, Tel: 09992935860; E-mail: shivani.dr04@gmail.com

Received date: Oct 03, 2015; Accepted date: Jan 02, 2016; Published date: Jan 05, 2016

Copyright: ( 2016 Malik S et al. This is an open-access article distributed under the terms of the Creative Commons Attribution License, which permits unrestricted use, distribution, and reproduction in any medium, provided the original author and source are credited.

\begin{abstract}
Phylloides tumor of breast is a rare tumor of fibroepithelial origin forming only about $0.3-0.5 \%$ of all breast malignancies. Malignant phylloids tumor is even more rarely encountered lesion of very unpredictable clinical outcome with high chances of recurrence and distant metastasis. Accurate preoperative pathological diagnosis is mandatory to allow correct planning of the surgical treatment which can be either wide excision or mastectomy with an intent to have a negative surgical margin of at least $1 \mathrm{cms}$. This rare and enigmatic tumor should be kept in mind while dealing with breast lumps. Here, we present a case of recurrent malignant phylloides tumor in a young female alongwith review of literature of this rare entity.
\end{abstract}

Aim and Objective: To present a case of recurrent malignant phylloides tumor in a young female alongwith its cytology images and to have a review of literature of this enigmatic entity.

Keywords: Malignant; Phylloides; Young; Recurrent; Female

\section{Introduction}

Phylloids tumor is a rare fibroepithelial lesion constituting only about $0.3-0.5 \%$ of the breast malignancies [1]. Malignant phylloides tumor is an entity of unpredictable behavior owing to its high chances of recurrence and distant metastasis [2]. Prompt diagnosis is mandatory for appropriate management.

\section{Case Presentation}

Our attention towards this topic was drawn by a case of recurrent malignant phylloids tumor in a young female who presented to our institution. A 22 year female presented with swelling of size approximately $4 \times 3 \mathrm{~cm}$ over a surgical scar mark on the right side of chest. The patient gave a history of right mastectomy for a breast lump around 3 years back. Her previous histopathological reports stated the lump to be a malignant phylloides tumor. On examination, the lump was hard with restricted mobility. The FNAC of the swelling over the scar was performed. Smears revealed hypercellular stromal fragments along with large number of highly pleomorphic, singly scattered, round to oval to spindle cells with retained cytoplasm at places. Numerous atypical mitotic figures were also evident. Diagnosis of malignant phylloides tumor was given which was confirmed on histopathology. The wide surgical excision of lump was done. The surgical margins were free of tumor infilteration on histopathological examination. No axillary lymphnodes were detected on palpation and imaging. The CT scan of chest and abdomen revealed no tumor metastasis. Patient has been administered two cycles of radiotherapy and is lost to follow up at present (Figure 1).

We would like to proceed with the discussion of this rare and aggressive malignancy, presenting the cytological images of the above said case.

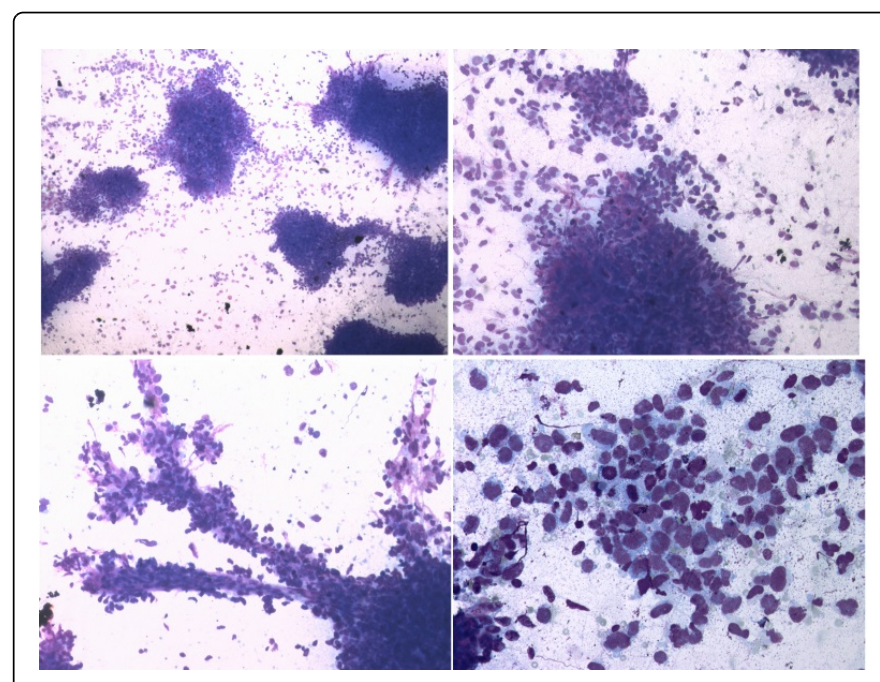

Figure 1: Phylloides Collage

\section{Discussion}

An extensive data search was made on the internet to gather all important information regarding this rare aggressive malignancy of variable and unpredictable behavior.

Phylloids tumor is a rare fibroepithelial tumor with the majority of them, been described as benign ( $35 \%$ to $64 \%$ ), with the remainder divided between the borderline and malignant subtypes [3]. Generally, it presents as a rapidly growing and clinically benign breast lump (usually $>3 \mathrm{cms}$ ) in females within the fourth or fifth decade of life. Only a few cases have been reported in young age group as was in our case. The average size of this tumor is around $4 \mathrm{cms}$, however approximately $20 \%$ attain a size larger than $10 \mathrm{cms}$. These tumors can 
reach sizes up to $40 \mathrm{~cm}$ in diameter. Mammography and ultrasonography constitute the chief imaging modalities. Wurdinger et al. show that round or lobulated shape, well-defined margins, heterogeneous internal structure, and nonenhancing internal septations are more common findings in phyllodes tumors than in fibroadenomas [4]. It has been seen that the chances of malignancy are greater in older patient however it should be kept in mind that even the young patient can have malignant phylloides tumor. As opposed to its benign counterpart, it is characterized by an unusually aggressive course and has been reported to recur locally and have the capacity for distant metastasis [2]. Komenaka et al. found the sensitivity of core needle biopsy to be $99 \%$ and negative predictive value and positive predictive value $93 \%$ and $83 \%$, respectively, for the diagnosis of phylloides tumor [5]. Histopathological features suggestive of malignancy include marked degree of stromal growth and atypia, $\geq 10$ mitotic figures per high power field and infilterative tumor margins.

Risk of recurrence is always associated with phylloids tumors, stated to be around $20-30 \%$ for the malignant one. In a retrospective study of 352 cases of PT from 1954 to 2005, positive margins, fibroproliferation rate, stromal pattern, stromal cellularity, frequency of mitosis, and necrosis were associated with an increase in local recurrence rates [6]. Distant metastasis, mostly through hematogenous route is found in approximately $25 \%$ of patients with malignant phylloides tumor and the dissemination is cheifly determined by the biological behavior of the tumor. The commonest sites for distant metastasis are the lungs $(66 \%)$, bones (28\%), and brain (9\%) and in rare instances, the liver and heart [7]. Metastasis to axillary lymph nodes occurred in only 2\% [8]. Various other malignancies as angiosarcoma, chondrosarcoma, osteosarcoma, liposarcoma, leiomyosarcoma, and rhabdomyosarcoma, are rarely encountered in malignant phyllodes tumors.

Main treatment modality includes wide excision with the aim of obtaining negative surgical margins of $>1 \mathrm{~cm}$. Since an excision with the required margins is often impossible in giant phyllodes tumors, mastectomy should be reserved for larger tumors and should be considered in recurrent tumors, especially of the malignant histotype [8]. Depending on the size of the breast and the location of the phyllodes tumor, mastectomy may also be required for tumors that are between 5 and $10 \mathrm{~cm}$ in diameter [9]. A retrospective review of malignant phylloides tumor in 478 patients was conducted to determine local control rates based on tumor size and type of surgery performed. Five-year local control rates were higher for patients treated with mastectomy compared with lumpectomy [6]. Belkacemi et al. reported that radiotherapy was associated with superior local control rate at 10 years, from $59 \%$ to $86 \%$ for both borderline and malignant phyllodes tumors [10]. Systemic chemotherapy and hormonal therapy is debatable in phyllodes tumor. To date there is no double blinded, multicenter study on this topic [11].

Regular follow up of the patient is necessary, 6 monthly during the first two years after treatment and then on yearly since this tumor is very unpredictable in recurrence and metastatic activity.

\section{Conclusion}

Phylloides tumor should be kept as a differential diagnosis while dealing with the breast lump patients. Furthemore, malignant phylloides is an aggressive breast neoplasm with high potential of recurrence and metastatic activity. So an appropriate diagnosis on time is mandatory for proper management.

\section{References}

1. Rowell MD, Perry RR, Hsiu J, Barranco SC (1993) Phyllodes tumors. The American Journal of Surgery 165: 376-79.

2. Parker SJ, Harries SA (2001) Phyllodes tumours. Postgrad Med J 77: 428-35.

3. Reinfuss M, Mitus J, Duda K, Stelmach A, Rys J, et al. (1996) The treatment and prognosis of patients with phyllodes tumor of the breast: an analysis of 170 cases. Cancer 77: 910-16.

4. Wurdinger S, Herzog, Fischer DR, Marx C, Raabe G, et al. (2005) Differentiation of phyllodes breast tumors from fibroadenomas on MRI. American Journal of Roentgenology 185: 1317-21.

5. Komenaka IK, El-Tamer M, Pile-Spellman E, Hibshoosh H (2003) Core needle biopsy as a diagnostic tool to differentiate phyllodes tumor from fibroadenoma. Archives of Surgery 138: 987-90.

6. Pezner RD, Schultheiss TE, Paz IB (2008) Malignant phyllodes tumor of the breast: local control rates with surgery alone. Int J Radiat Oncol Biol Phys 71: 710-13.

7. Jang JH, Choi MY, Lee SK, Kim S, Kim J, et al. (2012) Clinicopathologic risk factors for the local recurrence of phyllodes tumors of the breast. Ann Surg Oncol 19: 2612-17.

8. Asoglu O, Ugurlu MM, Blanchard K, Grant CS, Reynolds C, et al. (2004) Risk factors for recurrence and death after primary surgical treatment of malignant phylloides tumors. Ann Surg Oncol 11: 1011-7.

9. Baker RR (1990) Unusual lesions and their management. Surgical Clinics of North America 70: 963-75.

10. Belkacemi Y, Bousquet G, Marsiglia H, Ray-Coquard I, Magné N, et al. (2008) Phyllodes tumor of the breast. Int J Radiat Oncol Biol Phys 70: 492-500.

11. Roberts N, Runk DM (2015) Aggressive malignant phyllodes tumor. Int J Surg Case Rep 8: 161-5. 